$\mathrm{DE}$

M E D I C I N A

T R O P I C A L

$\mathrm{DE}$

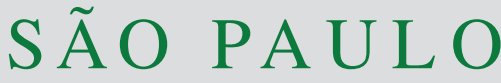

JOURNAL OF THE SÃO PAULO INSTITUTE OF TROPICAL MEDICINE

${ }^{1}$ Bhutan Ministry of Health, Royal Center for Disease Control, Thimphu, Bhutan

${ }^{2}$ Bhutan Ministry of Health, Department of Public Health, Vector Borne Disease Control Programme, Thimphu, Bhutan

${ }^{3}$ Bhutan Agriculture and Food Regulatory Authority, Thimphu, Bhutan

${ }^{4}$ Khesar Gyalpo University of Medical Science, Thimphu, Bhutan

${ }^{5}$ Thammasat University, Cholangiocarcinoma, Chulabhorn International College of Medicine, Center of Excellence in Pharmacology and Molecular Biology of Malaria, Pathumthani, Thailand

Correspondence to: Kesara NaBangchang

Thammasat University,

Cholangiocarcinoma, Chulabhorn International College of Medicine, Center of Excellence in Pharmacology and Molecular Biology of Malaria, Rangsit Campus, 99 Moo 18, Paholyothin Road, Rangsit 12121, Pathumthani, Thailand

E-mail: kesaratmu@yahoo.com

Received: 24 April 2019

Accepted: 31 July 2019

\section{Malaria elimination in Bhutan: asymptomatic malaria cases in the Bhutanese population living in malaria-risk areas and in migrant workers from India}

\author{
Sonam Wangchuk', Sonam Gyeltshen ${ }^{1}$, Kunzang Dorji', Tenzin Wangdi ${ }^{2}$, \\ Tobgyel Dukpa², Rinzin Namgay², Sithar Dorjee ${ }^{3,4}$, Tashi Tobgay ${ }^{4}$, Wanna \\ Chaijaroenkul $^{5}$, Kesara Na-Bangchang ${ }^{(15}$
}

\section{ABSTRACT}

In 2018, Bhutan reported 54 cases of malaria, of which six were indigenous, 14 introduced and 34 imported. Considering the continuous reduction in the number of indigenous cases, Bhutan plans to eliminate malaria by 2025 under the Bhutan Malaria Elimination Strategy. The study was conducted to assess the presence of asymptomatic plasmodial infection in both, Bhutanese population living in malaria-risk areas and in migrant workers to guide the elimination strategies. A cross-sectional study was conducted from April to May 2016 in 750 Bhutanese people and 473 migrant workers. Plasmodium falciparum and Plasmodium vivax infections were investigated by using a rapid diagnostic test (RDT) and the polymerase chain reaction (PCR). Prevalence of asymptomatic plasmodial infection based on PCR was $0.27 \%$ (95\% CI: $0.05-1.07 \%$ ) among Bhutanese people with a mean age of 43 years old. The proportions of males and females were $45 \%$ and $55 \%$, respectively. Among migrant workers, the prevalence of asymptomatic plasmodial infection was $0.42 \%$ (95\% CI: $0.07-$ $1.69 \%$ ) with a mean age of 30 years old. The majority of migrant workers were from the neighboring Indian State of West Bengal (57.51\%), followed by Assam (12.26\%). RDT in both study groups did not detect any plasmodial infection. The presence of a low prevalence of asymptomatic plasmodial infection indicates that the current elimination strategies and interventions are effective.

KEYWORDS: Malaria. Asymptomatic. Malaria elimination. Bhutan. PCR. Rapid Diagnostic Test (RDT).

\section{INTRODUCTION}

Globally, the incidence rate of malaria has decreased from 72 to 59 cases per 1,000 at risk inhabitants between 2010 and 2017, a 18\% reduction ${ }^{1}$. An estimated 219 million cases of malaria occurred worldwide in 2017 compared to 239 million cases in 2010. However, the estimates for 2015-2017 were almost similar, suggesting no progress in reducing the malaria burden during these last three years. In 2017 , malaria resulted in an estimated 435,000 deaths globally compared to 607,000 deaths in 2010 , with a $28 \%$ decrease in mortality ${ }^{1}$. This reduction was attributed to the availability of highly effective antimalarial drugs and long-lasting insecticide nets (LLINs), as well as a mutual investment to provide treatment and preventive measures to the people in need ${ }^{1-3}$. Most malaria cases were reported from the African World Health Organization (WHO) (92\%), followed by the South-East Asia Region WHO (5\%), and the Eastern Mediterranean Region WHO (2\%). Notably, 
the highest numbers of malaria cases and deaths (93\%) were reported from the African WHO, mostly in children under five years of age ${ }^{1}$. To accelerate progress in reducing the burden of malaria, WHO endorsed the Global Technical Strategy for Malaria 2016-2030 (GTS) which set a vision to eliminate malaria in 35 countries by 2030 and in at least ten countries by $2020^{1}$.

Bhutan has achieved a significant reduction of malaria incidence from 1,868 indigenous cases in 2006 to only six indigenous cases in $2018^{4-8}$. The geographical distribution of malaria infection has also decreased from 15 of the 20 districts in 2006 to only two districts in $2018^{4,6}$. The dramatic decline in malaria cases is believed to be due to the high coverage of LLINs, intensified surveillance and early diagnosis and treatment $t^{5,6,9,10}$. Bhutan plans to eliminate malaria by $2025^{\circ}$. However, imported and reintroduced cases along the international border with some Indian States remain a significant concern as seven of the seven Bhutanese districts (Chukha, Dagana, Pemagatshel, Samtse, Samdrup Jongkhar, Sarpang, and Zhemgang) share porous international borders with Assam, West Bengal, Arunachal Pradesh and Sikkim, in India. Among these States, Assam and West Bengal borders have intense cross-border activity, and most cases are reported in areas bordering Assam ${ }^{5,11,12}$. Percentages of imported malaria cases have increased from $79.7 \%$ of the total malaria cases detected in Bhutan in 2016 to $82.33 \%$ in 2017 , and $88.9 \%$ in $2018^{4,10,13}$.

In Bhutan, Plasmodium species that cause malaria are $P$. falciparum and P. vivax. Anopheles species recorded and considered as malaria vectors in Bhutan are $A n$. minimus, An. fluviatilis, An. dirus, An. pseudowillmori and An. culicifacies $^{13}$. However, no studies on vectors including their ecology and behaviors have been conducted in Bhutan. The primary malaria control intervention adopted in the country includes mandatory screening for plasmodial infections in any fever case, early detection and treatment, active case finding to detect foci of transmission, community awareness and education. In addition, vector control by the universal coverage of LLINs, indoor residual spraying (IRS), clearing bushes and avoiding stagnation of water in the surrounding have also been implemented. While malaria incidence has dramatically declined, there is not much information on asymptomatic reservoirs in the country. Some evidences suggest that a significant proportion of asymptomatic reservoirs are present in both, high and low transmission settings ${ }^{14,15}$. The diagnosis of asymptomatic plasmodial infections in people living in low transmission settings cannot be made by commonly used diagnostic methods, i.e., microscopic examination and rapid diagnostic tests (RDT) ${ }^{16}$. To achieve malaria elimination, it is essential to ascertain the burden of asymptomatic reservoirs in the population at risk, as well as in migrant workers from malaria-endemic countries, particularly India ${ }^{14,17}$, and proactively detect and treat asymptomatic plasmodial infections with effective antimalarial drugs ${ }^{18-21}$.

This study aimed to estimate the prevalence of asymptomatic plasmodial infections in the population living in at risk malaria areas in Bhutan, as well as in migrant workers from India. This information is essential to support the implementation of malaria elimination strategies in pursuit of elimination by $2025^{\circ}$.

\section{MATERIALS AND METHODS}

\section{Study area and sample size}

A cross-sectional survey was conducted to determine the prevalence of asymptomatic $P$. vivax and P. falciparum infections targeting populations living in risk areas for malaria in seven districts of Bhutan, i.e., Chukha, Dagana, Pemagatshel, Samtse, Sarpang Samdrup Jongkhar, and Zhemgang (Figure 1). The study period coincided with the peak of the malaria season in April to May 2016. Based on the records maintained by 16 health centers in the risk areas they consisted of 6,319 households and 28,583 people, accounting for approximately $4 \%$ of the country's population. For the estimation of the prevalence of asymptomatic plasmodial infections ( $P$. vivax and $P$. falciparum), catchment areas of two health centers in each of these districts located in malaria risk areas (based on the number of cases detected from 2011-2015) were intentionally selected ${ }^{22}$. From each selected health center, approximately four villages (primary sampling units) were randomly selected and from each selected village, a maximum of ten households (secondary sampling units) were selected using a systematic random sampling technique (based on data maintained by VDCP and health centers). When the selected village had less than ten households, additional village(s) were randomly sampled, from the eligible list of villages in the catchment area of the selected health centers. From each household, a single member in the eligible list of household members was randomly sampled based on the inclusion criteria: (i) individual residing in a household that received LLINs distributed by the Vector-Borne Disease Control program in 2014; (ii) had not been diagnosed or treated for malaria during the last 21 days (based on the maximum incubation period $^{23}$ ), (iii) aged $\geq 18$ years on the date of the survey, (iv) resident in the locality for $\geq 1$ years and (v) agreed to participate in the study.

Using a $95 \%$ confidence interval $(95 \% \mathrm{CI})$, an error of $2 \%$ and an anticipated asymptomatic prevalence of $5 \%$ 


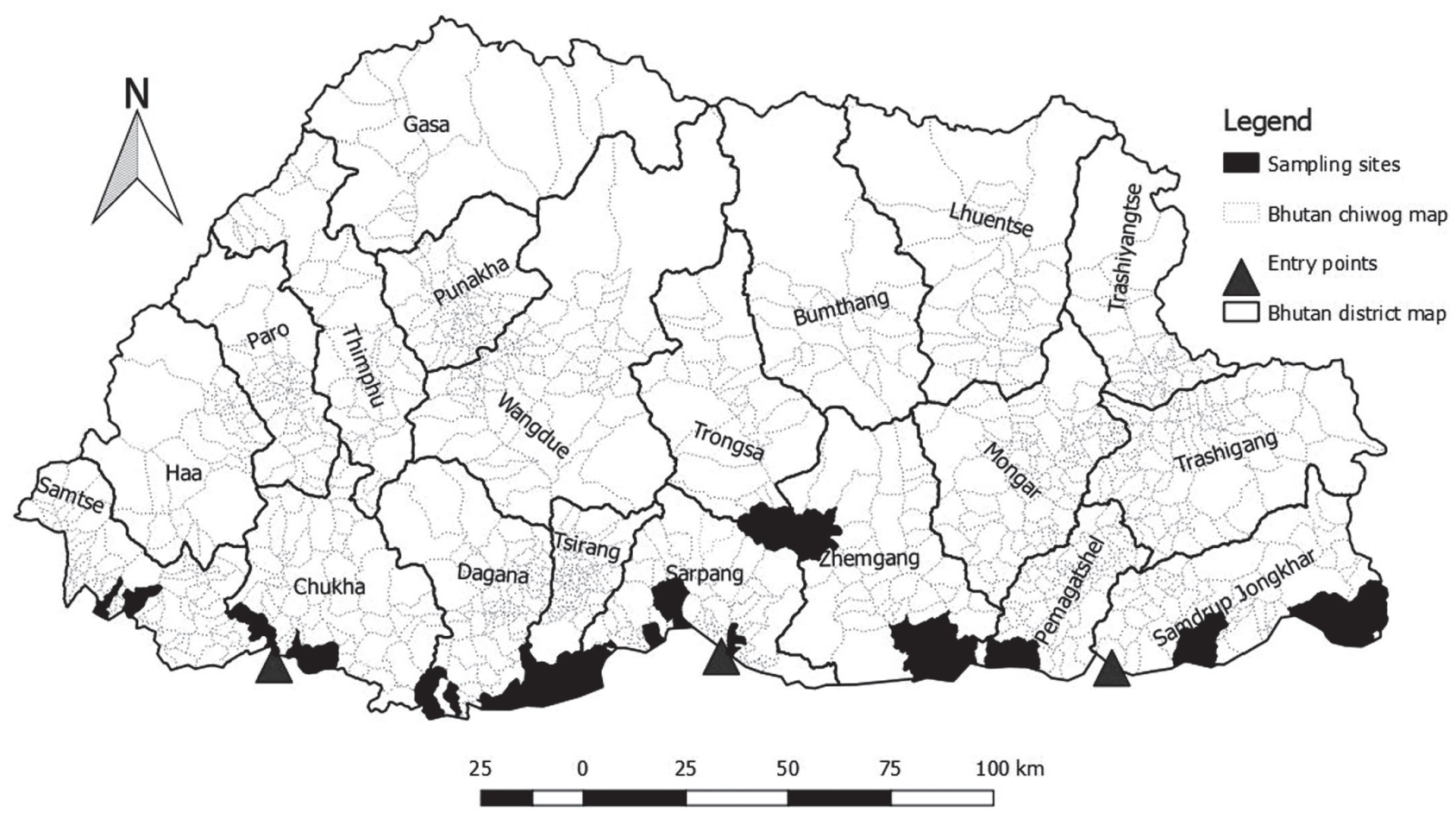

Figure 1 - Bhutan's chiwog map (small administrative units are in dotted lines) and the district map (bold lines) shows the sampling sites (shaded in black color) to estimate the prevalence of asymptomatic malaria in the community and in migrant workers at the three border entry points (triangles).

(the expected prevalence of $5 \%$ was assumed although previous small-scale studies reported a prevalence of asymptomatic plasmodial infections $<1 \%{ }^{5}$ ). The required sample size was 457 participants. To account for the loss and missing information (approximately 10\%), the sample size was rounded to 500 . Taking the maximum design effect of $1.5^{18}, 750$ individuals were included in the analysis using the formula $\mathrm{N}=(\mathrm{Z} \alpha / 2)^{2 * \mathrm{P}}(1-\mathrm{P}) * \mathrm{DEFF} / \mathrm{ME}^{2}$, where $\mathrm{N}$ is the sample size, $\mathrm{Z} \alpha / 2$ is the critical $\alpha$ level, $\mathrm{P}$ is the anticipated asymptomatic malaria prevalence, DEFF is the design effect, and ME is the marginal error.

Migrant workers were enrolled in three main entry points, i.e., Phuntsholing, Gelephu and Samdrup Jongkhar. All migrant workers underwent compulsory medical examination for entry into Bhutan. To this end, there were five registered private diagnostic centers in Phuntsholing, two in Gelephu and one in Samdrup Jongkhar. The sample size required for the estimation of the prevalence of asymptomatic malaria in this group of migrant workers was estimated using the same formula (without adjusting for the design effect DEFF assuming a within-private-diagnosticcenters variance of zero). Therefore, the estimated sample size for migrant workers entering Bhutan through these entry points was 500 individuals. Since over $50 \%$ of the workers entered through Phuntsholing, and around 50\% through Gelephu and Samdrup Jongkhar, 250 individuals were randomly sampled in Phuntsholing and 125 individuals in Gelephu and the same number in Samdrup Jongkhar.

Approval of the study protocol was obtained from the Research Ethics Board of Health (REBH), Ministry of Health, Royal Government of Bhutan (approval No. REBH/ Approval/2016/016). Written informed consent was obtained from the head of each household ( $\mathrm{HH})$ family. The interviewers explained the purpose, the risks and benefits of the study in the participant language and participation in the survey were voluntary. Written information on the survey was translated into Dzongkha for Bhutanese and Hindi for migrant workers and provided to all participants. Participants' demographics were collected using a structured questionnaire.

\section{Sample collection and testing}

Venous blood samples were collected from all the subjects for the RDT and three aliquots of $50 \mu \mathrm{L}$ were directly spotted onto filter papers (Whatman No. 3 MM, GE Healthcare UK Limited, Buckinghamshire, United Kingdom). Venous blood sample collection was chosen due to the difficulty to obtain adequate blood samples volumes by the finger-prick procedure as most farmers in the community had hard finger skins due to the type of work they do. Each filter paper (Dried Blood Spot, DBS) was air-dried at $25^{\circ} \mathrm{C}$ overnight, kept in 
a zip-lock plastic bag containing desiccants and stored at $25^{\circ} \mathrm{C}$ until it was sent to the laboratory and processed ${ }^{24,25}$.

The RDT used in the study was the FirstSign ${ }^{\mathrm{TM}}$ ParaView-2 (Unimed, Volmolenheide, Belgium) and the test was performed according to the manufacturer's instructions. This immuno-chromatography test detects $P$. falciparum specific histidine-rich protein-2 (HRP-2) and P. vivax specific $\mathrm{pLDH}$.

Genomic DNA was extracted from DBS samples using the QIAamp DNA Mini Kit (QIAGEN ${ }^{\circledR}$, Hilden, Germany) according to the manufacturer's instructions. The extracted DNA was stored at $-20{ }^{\circ} \mathrm{C}$ until use. Nested PCR assay was carried out as previously described ${ }^{26}$. DNA samples were amplified using species-specific primer pairs designed to amplify small subunit ribosomal ribonucleic acid (ssrRNA) genes of $P$. falciparum and $P$. vivax. The outer primers were rPLU 1: 5'TCAAAGATTAAGCCATGCAAGTGA3' and rPLU5: 5'CCTGTTGTTGCCTTAAACTCC3'. The primers used for nested PCR for $P$. falciparum were FA L1:5'TTAAACTGGTTTGGGAAAACCAAATATATT3' and FAL2: 5' ACACAATGAACTCAATCATGACTACCC GTC3' and for $P$. vivax VIV1: 5'CGCTTCTAGCTTAATC CACATAACTGATAC3' and VIV2:5' ACTTCCAAGCCG AAGCAAAGAAAGTCCTTA3. In brief, the primary amplification was carried out in a total volume of $25 \mu \mathrm{L}$ containing $1 \mathrm{X}$ buffer, $3.5 \mathrm{mM} \mathrm{MgCl}_{2}, 0.2 \mathrm{mM}$ dNTPs, $0.2 \mu \mathrm{M}$ primers and $1 \mathrm{U}$ Taq DNA-polymerase, $2.0 \mu \mathrm{L}$ of extracted DNA (25 ng), and nuclease-free water in a T100 ${ }^{\mathrm{TM}}$ Thermal cycler (BioRaid, Singapore). The nested amplification was carried out in a $20 \mu \mathrm{L}$ reaction volume containing the same reaction mixture, $P$. falciparum and $P$. vivax species-specific primer, and $1 \mu \mathrm{L}$ of the $\mathrm{PCR}$ product from the primary amplification. Thermal cycling conditions for the first round of amplification were as follows: initial denaturation at $94{ }^{\circ} \mathrm{C}$ for $1 \mathrm{~min}$, followed by 35 cycles of $30 \mathrm{~s}$ at $94{ }^{\circ} \mathrm{C}, 60 \mathrm{~s}$ for $55^{\circ} \mathrm{C}$ and $60 \mathrm{~s}$ at $72{ }^{\circ} \mathrm{C}$, followed by a final extension of $10 \mathrm{~min}$ at $72{ }^{\circ} \mathrm{C}$. The cycling conditions for the second PCR were similar, excepting for the annealing temperature that was set at $58{ }^{\circ} \mathrm{C}$. Negative and positive controls were included in each experiment. Amplicons were separated by $2 \%$ agarose gel electrophoresis at $100 \mathrm{~V}$ with the molecular weight marker 100 bp DNA ladder (Bioline, Memphis, USA). The presence or absence of different Plasmodium species was confirmed by the presence of a 205 bp amplicon for P. falciparum and a 117 bp amplicon for $P$. vivax.

\section{Statistical analysis}

All data were entered into the Epi Info ${ }^{\mathrm{TM}}$ version 7.2.0.1, and statistical analysis was performed using the Stata version 14.0 (Stata Statistical Software, Release 14: Stata Corp, College Station, TX, USA).

The frequencies of asymptomatic malaria caused by the two species are summarized as proportions, with $95 \%$ confidence intervals (95\% CI).

\section{RESULTS}

\section{Demographic characteristics of the participants}

\section{Participants from malaria risk areas}

A total of 750 participants were enrolled from 75 villages in malaria risk areas of the seven districts of Bhutan. The participants' age ranged from 18 to 84 years, with a mean age of 45 years old. The male to female participants' ratio was $1: 1.2$. The participants were predominantly farmers (91.87\%), and the remaining participants were students, business people, government/private employees and religious practitioners.

\section{Migrant workers}

From a total of 500 migrant workers, 473 were enrolled in five private diagnostic centers (Druk Diagnostic, Garuda Diagnostic Center, New Life Diagnostic Service, Phuntsholing Diagnostic Center, and Samdrup Jongkhar Diagnostic Center) at the three border entry points (Phuntsholing, Gelephu, and Samdrup Jongkhar). All of them were males with age ranging from 18 to 66 years (mean of 30 years). Most were from West Bengal (57.51\%), followed by Assam (12.26\%) and the remaining were from Bihar, Uttar Pradesh, Himachal Pradesh, Manipur and Orissa. Information on the States in India where they used to live was not obtained from a significant part of the migrant workers $(27.27 \%)$.

\section{Detection of $P$. falciparum and $P$. vivax parasitemia by RDT and PCR}

\section{Participants from malaria risk areas}

From the total of 750 samples collected from villagers, $11(1.46 \%)$ samples were not tested by RDT as one of the clusters had inadequate RDT. All of the 739 samples showed negative results by RDT. Based on the PCR analysis, 2 of the 739 samples were positive $(0.27 \%)$ for $P$. vivax and none for $P$. falciparum. The estimated prevalence of asymptomatic plasmodial infections in the studied areas was $0.27 \%(95 \%$ CI: 0.05-1.07\%) (Table 1). Positive samples were obtained from Chukha district, one sample from Lingden village (32 years old male) and another sample from Kothilina villager (a 38 years old male). All positive cases were treated according to the national protocol. 
Table 1 - Detection of $P$. falciparum and $P$. vivax infections by RDT and PCR in participants from malaria risk areas (750 cases) and migrant workers from the three border entry points (473 cases). Data are presented as numbers (percentages) and $95 \%$ confidence intervals $(\mathrm{Cl})$ when appropriate.

\begin{tabular}{|c|c|c|c|c|}
\hline & \multicolumn{2}{|c|}{ Malaria risk areas $(\mathrm{N}=750)$} & \multicolumn{2}{|c|}{ Migrant workers $(\mathrm{N}=473)$} \\
\hline & $\begin{array}{c}\text { RDT } \\
(\mathrm{n}=739)\end{array}$ & $\begin{array}{c}\text { PCR } \\
(n=735)\end{array}$ & $\begin{array}{c}\text { RDT } \\
(n=473)\end{array}$ & $\begin{array}{c}\text { PCR } \\
(n=473)\end{array}$ \\
\hline P. falciparum & $0(0)$ & $0(0)$ & $0(0)$ & $0(0)$ \\
\hline P. vivax & $0(0)$ & $\begin{array}{c}2(0.27 \%) \\
\text { (95\% Cl: } 0.05-1.07 \%)\end{array}$ & $0(0)$ & $\begin{array}{c}2(0.42 \%) \\
\text { (95\% Cl: } 0.07-1.69 \%)\end{array}$ \\
\hline
\end{tabular}

\section{Migrant workers}

From the total of 473 migrant worker samples, all of them showed negative results by RDT, and only 2 samples showed positive results for P. vivax PCR. One P. vivax positive infection was from Assam (a 21 years old male), and another one was from West Bengal (a 27 years old male). The estimated prevalence of asymptomatic plasmodial infections in this group of migrant workers was 0.42\% (95\% CI: 0.07-1.69\%) (Table 1).

\section{DISCUSSION}

Asymptomatic and submicroscopic parasitemia reservoirs can sustain the continuous transmission of malaria even in low transmission settings if they are not accurately detected by a highly sensitive detection method and immediately treated by an effective antimalarial drug ${ }^{14-16}$. Therefore, it is essential to address the asymptomatic and submicroscopic parasitemia in the population to achieve malaria elimination ${ }^{16}$. Most countries with low transmission settings and striving to eliminate malaria, including Bhutan, have observed a large proportion of asymptomatic plasmodial infections, particularly of cases with submicroscopic parasitemia ${ }^{3,20,21,27}$.

This study is the first report on asymptomatic plasmodial infections that represented all malaria risk areas in Bhutan ${ }^{5}$. PCR was used in addition to RDT for malaria detection. This study was conducted during the premonsoon season (April-May 2016) coinciding with the peak of the malaria season in the country, to maximize the detection of asymptomatic reservoirs. Results suggest that the prevalence of asymptomatic and submicroscopic parasitemia cases could be very low $(0.27 \%)$ in the malaria risk areas in Bhutan. The two positive cases detected were P. vivax infections. Although this study was conducted in 2016, we believe that the prevalence of asymptomatic plasmodial infections remains unchanged. The prevention and control program in these areas have been maintained at the same level. There was no significant political and socio-economic changes in the country. The malaria cases decreased in 2018 compared to 2016. Nevertheless, Bhutan needs to strengthen and sustain an optimal surveillance, even though the prevalence of asymptomatic plasmodial infections was very low. The following reasons support this proposal: (i) Bhutan shares open borders with endemic areas of Indian States (Assam and West Bengal), (ii) The climate conditions in these border areas include other high-risk areas with tropical and subtropical climate and (iii) there is abundance of vector mosquito populations in these areas. Results indicate that the current elimination strategies and interventions have been effective in interrupting malaria transmission in populations at risk, and asymptomatic and submicroscopic parasitemia cases are an insignificant source of malaria transmission in Bhutan. The observation is in agreement with previous reports from Sri Lanka ${ }^{28,29}$, Iran $^{30}$, Soloman Islands ${ }^{31}$ and Paraguay ${ }^{32}$. All these reports suggest the reduction of cases of asymptomatic and submicroscopic parasitemia malaria with decreased symptomatic malaria cases in the population at risk. The absence of asymptomatic plasmodial infections in Bhutan Has been previously reported ${ }^{5}$, but the study was limited to only four risk areas of the two districts bordering the Assam State of India (Sarpang and Samdrup Jongkhar). Besides, the method used for the detection of asymptomatic plasmodial infections was the RDT, which has poor sensitivity compared to $\mathrm{PCR}^{3,5,19-21}$.

A low prevalence of asymptomatic plasmodial infections was expected in Bhutan due to its low malaria transmission sustained over a long period. This could be attributed to the country's relentless effort in ensuring preventive measures such as the provision of LLINs and IRS, as well as the policy to perform the compulsory screening of all fever cases in malaria risk areas for early detection and treatment of the disease. Bhutan launched active case finding strategies targeting asymptomatic plasmodial infections as early as 2012. For the preparation towards the malaria elimination, the program included reactive case detection methods for the identification of parasite transmission foci, and proactive case detection methods in the screening of all migrant workers at border districts ${ }^{10,33-36}$. 
The LLIN's coverage exceeded 90\% since 2006, and successive surveys on indicators have also reported very high coverages ${ }^{13,34,35}$. Malaria cases were also dramatically reduced from 465 cases in 2010 to 104 cases in 2015, with $65 \%$ of imported cases. Notably, $54 \%$ of the imported cases were non-national ${ }^{13}$. Among the indigenous cases, $74 \%$ were residing close to the Bhutan-India border, suggesting an active transmission in pockets of border areas due to the continuous movement of people across the border ${ }^{12}$. The genetic analyses have also suggested an active transmission in border areas, as the same genotype was found in both, Bhutanese and non-national (Indian) people ${ }^{37}$.

Most of the migrant workers were from Indian States of West Bengal (57.51\%), followed by Assam (12.26\%). The remaining cases were from Bihar, Uttar Pradesh, Himachal Pradesh, Manipur and Orissa. These figures were in agreement with previous reports on the high number of imported cases from West Bengal, Assam and Bihar in the past years due to its proximity ${ }^{38-40}$ and the majority of migrant workers coming from these two regions. All of these States are endemic to malaria and responsible for the highest number of malaria cases and deaths in India ${ }^{11,41}$. High prevalences of asymptomatic malaria cases have also been reported in a tribal population in Eastern and North East India $^{14,41}$. Contrary to our expectations, despite the high incidence of malaria cases in these States, a low prevalence of asymptomatic malaria was found in these imported cases $(0.48 \%)$. Mass malaria screening of migrant workers has been routinely implemented, annually, by the Vector-Borne Disease Control Program (VDCP) in different hydropower project sites in which very low prevalences of asymptomatic malaria cases were reported in Bhutan. Although a regular screening process at the point of entry is mandatory, the current RDT may not be sufficient to prevent asymptomatic carriers from entering Bhutan indicating the need for more regular and strengthened surveillance systems to monitor this population.

In 2014, VDCP screened 4,689 migrant workers in various hydropower projects sites also including Bhutanese people and only three $P$. vivax cases $(0.06 \%)$ were detected. In 2015 , only one P. vivax and one P. falciparum $(0.05 \%)$ cases were detected from 3,754 migrant workers screened with no asymptomatic case detected from 1,534 people screened in $2016^{39,40}$. However, the method used for the screening at these sites was microscopy and RDTs. Based on PCR analysis, absence of asymptomatic malaria cases has also been reported in the malaria-endemic area of Assam State $^{42}$. The low prevalence among migrant workers may also be attributed to the intensified malaria prevention and control activities in India ${ }^{43}$. The forest and illegal population dwelling inhabiting the international border may be greatly affected by malaria, but they may not be among the screened migrant workers. Only migrant workers moving for longer periods in Bhutan, but not daily workers who move on a daily basis were screened for malaria. According to the annual report of National Vector Borne Disease Control Program of India, the number of malaria cases decreased from 55,793 in 2012 to 26,240 in 2016 in West Bengal (a 47\% reduction) and from 29,999 in 2012 to 6,948 in 2016 in Assam (a 23\% reduction) ${ }^{44}$. The endemicity greatly varies within areas in these States ${ }^{44}$. Based on the results of this study, the asymptomatic reservoirs may not be a significant concern or barrier to the Bhutan's malaria elimination effort. However, a continuous high LLIN's coverage, special attention to the screening of migrant workers at border points and periodic mass screening at hydropower projects using more sensitive malaria detection methods such as RDT and PCR is required to eliminate any potential source of infection represented by both symptomatic and asymptomatic cases. To achieve Bhutan effort of malaria elimination in 2020, the current strategy of screening migrant workers at entry points and hydropower projects should be continued along with supportive control measures.

Low-density infections are common in asymptomatic individuals. Both, microscopy and RDT usually miss detection of infections when parasite densities are low $(<10$ parasites/ $\mu \mathrm{L})$. One of the most used RDT, is based on the capture of the parasite antigen by monoclonal antibodies ( $P$. falciparum specific HRP-2 and $P$. vivax specific pLDH). The test is very convenient, but is expensive. In addition, it can result in false-positives with variations of sensitivity and specificity. Nested PCR targeting the ssrRNA genes is the most sensitive and specific method, but it is also expensive, requiring an amplification infrastructure, equipment and reagents. Besides, it is a relatively sophisticated and time-consuming procedure, which may not be applicable to malaria diagnosis in remote areas. On the other hand, the detection limit of nested-PCR and nRT-PCR are as low as 5-10 and 22 parasites/ $\mu \mathrm{L}$ of blood, respectively ${ }^{45,46}$. Therefore, it is an important technique to investigate low parasite density in asymptomatic malaria patients.

\section{CONFLICT OF INTERESTS}

The authors declare that there are no conflict of interests

\section{ACKNOWLEDGMENTS}

The study was supported by the Vector Borne Disease Control Program under the Department of Public Health, 
Ministry of Health through Global Fund project. KN and WC were supported by Thammasat University, Thailand (Center of Excellence in Pharmacology and Molecular Biology of Malaria and Cholangiocarcinoma), National Research Council of Thailand.

\section{REFERENCES}

1. World Health Organization. The world malaria report 2018. Geneva: WHO; 2018.

2. World Health Organization. The world malaria report 2015. Geneva: WHO; 2015.

3. Pava Z, Burdam FH, Handayuni I, Trianty L, Utami RA, Tirta YK, et al. Submicroscopic and asymptomatic Plasmodium parasitaemia associated with significant risk of anaemia in Papua, Indonesia. PLoS One. 2016;11:e0165340.

4. Bhutan. Ministry of Health. Annual health bulletin. Thimphu: Ministry of Health; 2018.

5. Wangdi K, Gatton ML, Kelly GC, Clements AC. Prevalence of asymptomatic malaria and bed net ownership and use in Bhutan, 2013: a country earmarked for malaria elimination. Malar J. 2014;13:352.

6. Wangdi K, Kaewkungwal J, Singhasivanon P, Silawan T, Lawpoolsri S, White NJ. Spatio-temporal patterns of malaria infection in Bhutan: a country embarking on malaria elimination. Malar J. 2011;10:89.

7. Wangdi K, Singhasivanon P, Silawan T, Lawpoolsri S, White NJ, Kaewkungwal J. Development of temporal modelling for forecasting and prediction of malaria infections using timeseries and ARIMAX analyses: a case study in endemic districts of Bhutan. Malar J. 2010;9:251.

8. Yangzom T, Gueye CS, Namgay R, Galappaththy GN, Thimasarn $\mathrm{K}$, Gosling R, et al. Malaria control in Bhutan: case study of a country embarking on elimination. Malar J. 2012;11:9.

9. Bhutan. Ministry of Health. Vector-Borne Disease Control Programme. Bhutan National Malaria Elimination Strategy Plan 2015-2020. Gelephu: Ministry of Health; 2014.

10. West N, Gyeltshen S, Dukpa S, Khoshnood K, Tashi S, Durante A, et al. An evaluation of the National Malaria Surveillance System of Bhutan, 2006-2012 as it approaches the goal of malaria elimination. Front Public Health. 2016;4:167.

11. Das A, Anvikar AR, Cator LJ, Dhiman RC, Eapen A, Mishra N, et al. Malaria in India: the center for the study of complex malaria in India. Acta Trop. 2012;121:267-73.

12. Wangdi K, Gatton ML, Kelly GC, Clements AC. Cross-border malaria: a major obstacle for malaria elimination. Adv Parasitol. 2015;89:79-107.

13. World Health Organization. World malaria report 2016. Geneva: WHO; 2016.

14. Ganguly S, Saha P, Guha SK, Biswas A, Das S, Kundu PK, et al.
High prevalence of asymptomatic malaria in a tribal population in eastern India. J Clin Microbiol. 2013;51:1439-44.

15. Tadesse FG, Slater HC, Chali W, Teelen K, Lanke K, Belachew $\mathrm{M}$, et al. The relative contribution of symptomatic and asymptomatic Plasmodium vivax and Plasmodium falciparum infections to the infectious reservoir in a low-endemic setting in Ethiopia. Clin Infect Dis. 2018;66:1883-91.

16. Lin JT, Saunders DL, Meshnick SR. The role of submicroscopic parasitemia in malaria transmission: what is the evidence? Trends Parasitol. 2014;30:183-90.

17. Mukhopadhyay DK, Basu SS, Roy D, Das N, Akbar F, Sarkar GN. Netting the malaria menace: distribution and utilization of long-lasting insecticidal net in a malaria endemic area in Bankura, West Bengal. J Vector Borne Dis. 2016;53:23-9.

18. Golassa L, Baliraine FN, Enweji N, Erko B, Swedberg G, Aseffa A. Microscopic and molecular evidence of the presence of asymptomatic Plasmodium falciparum and Plasmodium vivax infections in an area with low, seasonal and unstable malaria transmission in Ethiopia. BMC Infect Dis. 2015;15:310.

19. Lo E, Zhou G, Oo W, Afrane Y, Githeko A, Yan G. Low parasitemia in submicroscopic infections significantly impacts malaria diagnostic sensitivity in the highlands of Western Kenya. PloS One. 2015;10:e0121763.

20. Tadesse FG, Pett H, Baidjoe A, Lanke K, Grignard L, Sutherland C, et al. Submicroscopic carriage of Plasmodium falciparum and Plasmodium vivax in a low endemic area in Ethiopia where no parasitaemia was detected by microscopy or rapid diagnostic test. Malar J. 2015;14:303

21. Motshoge T, Ababio GK, Aleksenko L, Read J, Peloewetse E, Loeto M, et al. Molecular evidence of high rates of asymptomatic P. vivax infection and very low P. falciparum malaria in Botswana. BMC Infect Dis. 2016;16:520.

22. Bhutan. Ministry of Health. Vector-borne Disease Control Programme. Annual malaria report 2015. Gelephu: Ministry of Health; 2015 .

23. South Africa. Department of Health. National guidelines for the treatment of malaria, South Africa, 2018. Pretoria: Department of Health; 2018.

24. Singh B, Bobogare A, Cox-Singh J, Snounou G, Abdullah MS, Rahman HA. A genus- and species-specific nested polymerase chain reaction malaria detection assay for epidemiologic studies. Am J Trop Med Hyg. 1999;60:687-92.

25. Singh B, Cox-Singh J, Miller AO, Abdullah MS, Snounou G, Rahman HA. Detection of malaria in Malaysia by nested polymerase chain reaction amplification of dried blood spots on filter papers. Trans R Soc Trop Med Hyg. 1996;90:519-21.

26. Lee WC, Chin PW, Lau YL, Chin LC, Fong MY, Yap CJ, et al. Hyperparasitaemic human Plasmodium knowlesi infection with atypical morphology in peninsular Malaysia. Malar J. 2013;12:88. 
27. Roh ME, Oyet C, Orikiriza P, Wade M, Kiwanuka GN, MwangaAmumpaire J, et al. Asymptomatic Plasmodium infections in children in low malaria transmission setting, Southwestern Uganda. Emerg Infect Dis. 2016;22:1494-8.

28. Rajakaruna RS, Alifrangis M, Amerasinghe PH, Konradsen F. Pre-elimination stage of malaria in Sri Lanka: assessing the level of hidden parasites in the population. Malar J. 2010;9:25.

29. Fernando SD, Abeyasinghe RR, Galappaththy GN, Rajapaksa LC. Absence of asymptomatic malaria infections in previously high endemic areas of Sri Lanka. Am J Trop Med Hyg. 2009;81:7637.

30. Zoghi S, Mehrizi AA, Raeisi A, Haghdoost AA, Turki H, Safari $\mathrm{R}$, et al. Survey for asymptomatic malaria cases in low transmission settings of Iran under elimination programme. Malar J. 2012;11:126.

31. Atkinson JA, Johnson ML, Wijesinghe R, Bobogare A, Losi L, O'Sullivan M, et al. Operational research to inform a subnational surveillance intervention for malaria elimination in Solomon Islands. Malar J. 2012;11:101.

32. Barrios ED, Russomando G, Puerto FD. Absence of asymptomatic cases of malaria in a historically endemic indigenous locality of the Department of Caaguazu, Paraguay: moving toward elimination. Rev Soc Bras Med Trop. 2016;49:637-40.

33. Sturrock HJ, Hsiang MS, Cohen JM, Smith DL, Greenhouse B, Bousema T, et al. Targeting asymptomatic malaria infections: active surveillance in control and elimination. PLoS Med. 2013; 10:e1001467.

34. Wangdi K, Banwell C, Gatton ML, Kelly GC, Namgay R, Clements AC. Malaria burden and costs of intensified control in Bhutan, 2006-14: an observational study and situation analysis. Lancet Glob Health. 2016;4:e336-43.

35. Wangdi K, Banwell C, Gatton ML, Kelly GC, Namgay R, Clements AC. Development and evaluation of a spatial decision support system for malaria elimination in Bhutan. Malar J. 2016;15:180.

36. Smith Gueye C, Sanders KC, Galappaththy GN, Rundi C, Tobgay

$\mathrm{T}$, Sovannaroth $\mathrm{S}$, et al. Active case detection for malaria elimination: a survey among Asia Pacific countries. Malar J. 2013;12:358.
37. Wangchuk S, Drukpa T, Penjor K, Peldon T, Dorjey Y, Dorji K, et al. Where chloroquine still works: the genetic make-up and susceptibility of Plasmodium vivax to chloroquine plus primaquine in Bhutan. Malar J. 2016;15:277.

38. Bhutan. Ministry of Health. Vector-Borne Disease Control Programme. Report on screening of malaria of migrant laboures at PHPA I \& PHPA II (15.07.2018 to 26.7.2018). Gelephu: Ministry of Health; 2018.

39. Dukpa S. Report of mass screening for malaria: Punatsangchu Projects (1/7/14 - 2/8/14, August, 2014). Gelephu: Ministry of Health, Vector-Borne Disease Control Program; 2013.

40. Bhutan. Ministry of Health. Vector-Borne Disease Control Programme. Report of mass screening for malaria: Punatsangchu Projects (13th June - 8th August. 2015). Gelephu: Ministry of Health, Vector-Borne Disease Control Program; 2015.

41. Sharma J, Dutta P, Khan SA. Epidemiological study of malaria cases in North East region of India. Indian J Med Microbiol. 2016;34:261-2.

42. Das NG, Dhiman S, Talukdar PK, Goswami D, Rabha B, Baruah I, et al. Role of asymptomatic carriers and weather variables in persistent transmission of malaria in an endemic district of Assam, India. Infect Ecol Epidemiol. 2015;5:25442.

43. Kakkilaya BS. Malaria in India. [cited 2019 Aug 6]. Available from: https://www.malariasite.com/malaria-india/

44. India. National Vectorborne Disease Control Program. Malaria situation. [cited 2019 Aug 20]. Available from: https://www. nvbdcp.gov.in/WriteReadData/1892s/75840419771565787319. pdf

45. Harris I, Sharrock WW, Bain LM, Gray KA, Bobogare A, Boaz $\mathrm{L}$, et al. A large proportion of asymptomatic Plasmodium infections with low and sub-microscopic parasite densities in the low transmission setting of Temotu Province, Solomon Islands: challenges for malaria diagnostics in an elimination setting. Malar J. 2010; 9:254.

46. Adams M, Joshi SN, Mbambo G, Mu AZ, Roemmich SM, Shrestha $\mathrm{B}$, et al. An ultrasensitive reverse transcription polymerase chain reaction assay to detect asymptomatic low-density Plasmodium falciparum and Plasmodium vivax infections in small volume blood samples. Malar J. 2015;14:520. 